\title{
On the Ranks of Homotopy Groups of a Space
}

\author{
By
}

\section{Kouyemon IRIYE}

For a simply connected space $X$ of finite type and a prime $p$ we define two power series

$$
P_{\pi}(X)=\sum\left(\operatorname{dim} \pi_{n}(X) \otimes Z_{p}\right) \cdot t^{n}
$$

and

$$
P_{H}(X)=\sum\left(\operatorname{dim} H_{n}\left(\Omega X ; Z_{p}\right)\right) \cdot t^{n} \text { 。 }
$$

Let $R_{\pi}(X)$ and $R_{H}(X)$ (or simply $R_{\pi}$ and $R_{H}$ ) be the radiuses of convergence of $P_{\pi}(X)$ and $P_{H}(X)$ respectively. Henn [2] proved

Theorem 1. Let $X$ be a simply connected space of finite type and $p$ be a prime. Then $R_{\pi} \geqq \min \left\{R_{H}, C_{p}\right\}$, where $C_{p}$ is a constant depending only on $p$ and $1 \geqq C_{2} \geqq 1 / 2,1 \geqq C_{p} \geqq 3^{-1 /(2 p-3)}$ for an odd prime $p$.

and conjectured

Conjecture. If $X$ is a simply connected finite complex then $R_{\pi}(X)$ $=R_{H}(X)$.

In this paper we give a following partial answer to the above.

Theorem 2. $\min \left\{1, R_{\pi}\right\} \leqq R_{H}$ for all simply connected spaces of finite type.

Thus Theorems 1 and 2 imply

Corollary. Let $X$ be a simply connected space of finite type and $p$ be a prime. If $R_{H}(X) \leqq C_{p}$, then $R_{\pi}(X)=R_{H}(X)$.

\footnotetext{
*This research was partially supported by the Alexander von Humboldt Foundation.

Communicated by N. Shimada, August 25, 1986.

Department of Mathematics, Kyoto University, Kyoto 606, Japan.
} 
Remark. (1) Let $R_{\pi_{*}\left(x ; Z_{p}\right)}$ be the radius of convergence of the power series $\sum\left(\operatorname{dim}\left(\pi_{n}\left(X ; Z_{p}\right) \otimes Z_{p}\right)\right) \cdot t^{n}$. Using the short exact sequence

$$
0 \rightarrow \pi_{n}(X) \otimes Z_{p} \rightarrow \pi_{n}\left(X ; Z_{p}\right) \rightarrow \pi_{n-1}(X) * Z_{p} \rightarrow 0
$$

for $n \geqq 4$, we see that $R_{\pi}(X)=R_{\pi_{*}\left(X ; z_{p}\right)}$. So his statement ([2], Theorem 1) is equivalent to us.

(2) In [2] he proved only that $C_{p} \geqq 1 / 2$, but it is not difficult to get the above estimate for an odd prime.

Example. Consider the suspension space of a connected space of finite type. By the Bott-Samelson theorem [1] $H_{*}\left(\Omega \Sigma X ; Z_{p}\right) \cong$ $T\left(H_{*}\left(X ; Z_{p}\right)\right)$, where $T$ is the tensor algebra functor. If $\operatorname{dim}$ $\tilde{H}_{*}\left(X ; Z_{p}\right)>1$, then by Theorem $2 \quad R_{\pi}(\Sigma X) \leqq R_{H}(\Sigma X)<1$. This result implies the existence of an infinite family of integers $\left\{q_{i}\right\}$ and $C>1$ such that $\operatorname{dim} \pi_{q_{i}}(\Sigma X) \otimes Z_{p} \geqq C^{q_{i}}$.

The author is grateful to the hospitality of the Mathematisches Institut der Universität Heidelberg, at which this research was done. He also would like to thank R. Schön and H. -W. Henn for helpful conversation.

\section{Proof of Theorem 2.}

From now on we deal with only the case that $p=2$. Because for an odd prime the following arguments work quite similarly. So $H_{*}(X)$ stands for $H_{*}\left(X ; Z_{2}\right)$.

For a space $X$ of finite type we define a power series

$$
P(X)=\sum\left(\operatorname{dim} H_{n}(X)\right) \cdot t^{n} \text {. }
$$

For a power series $f$ we denote by $\mathrm{r}(f)$ its radius of convergence. Thus $P_{H}(X)=P(\Omega X)$ and $R_{H}(X)=\mathrm{r}(P(\Omega X))$. Theorem 2 is deduced from the following theorem.

Theorem 3. Let $X$ be a simple space of finite type. Then $\min \left\{1, \mathrm{r}\left(P_{\pi}(X)\right)\right\} \leqq \mathrm{r}(P(X))$.

Now we prove Theorem 3. Let $X$ be a simple space of finite 
type. $\left\{X_{n}\right\}$ denotes the Postonikov decomposition of $X$. In particular

$$
\pi_{i}\left(X_{n}\right)= \begin{cases}\pi_{i}(X) & \text { for } i \leqq n \\ 0 & \text { for } i>n\end{cases}
$$

and there is a fibration

$$
K\left(\pi_{n}(X), n\right) \rightarrow X_{n} \rightarrow X_{n-1} \text {. }
$$

Notice that, since $X$ is simple, the local coefficient system over $X_{n-1}$ associated with the Serre fibration (4) is trivial. Then the Serre spectral sequence associated with (4) induces the following relation among the three power series:

$$
P\left(X_{n-1}\right) \cdot P\left(K\left(\pi_{n}(X), n\right)\right) \geqq P\left(X_{n}\right),
$$

where the sign $\geqq$ signifies that each coefficient of the first power series is equal to or larger than the corresponding coefficient to the second. Since $X_{0}=*$ and $P\left(X_{n}\right) \equiv P(X) \bmod \left(t^{n+1}\right)$, the above inequations for $n \geqq 1$ induce the inequation

$$
\Pi_{n \geq 1} P\left(K\left(\pi_{n}(X), n\right)\right) \geqq P(X) .
$$

For ' a finitely generated abelian group $\pi, P(K(\pi, n))$ is wellknown by Serre.

Theorem 6. ([3]) For $m \geqq 1$ and $q \geqq 0$,

$$
P\left(K\left(Z_{2^{m}}, q+1\right)\right)=\Pi_{h_{1} \geqq \cdots \geqq h_{q} \geqq 0}\left(1-2^{h^{h_{1}}+\cdots+2^{h}+1}\right)^{-1} \geqq P(K(Z, q+1)) \text {. }
$$

Put

$$
\begin{aligned}
& m(q)=\operatorname{dim} \pi_{q}(X) \otimes Z_{2} \\
& M(q)=\max \{m(1), \cdots, m(q)\}, \\
& a(n, q)=\#\left\{h_{1} \geqq \cdots \geqq h_{q} \geqq 0 ; 2^{h_{1}}+\cdots+2^{h_{q}}=n\right\}, \\
& a(n)=\sum_{q \geqq 0} a(n, q)
\end{aligned}
$$

and

$$
b(n)=\sum_{q \geq 0} a(n-1, q) M(q+1) .
$$

Then by (5) and Theorem 6 we have

$$
\begin{aligned}
\Pi_{n \geq 1}\left(1-t^{n}\right)^{-b(n)} & =\Pi_{q \geq 0, n \geq 1}\left(1-t^{n}\right)^{-a(n-1, q) M(q+1)} \\
& \geqq \Pi_{q \geq 0, n \geq 1}\left(1-t^{n}\right)^{-a(n-1, q) m(q+1)}
\end{aligned}
$$




$$
\begin{aligned}
& =\Pi_{q \geqq 0} \Pi_{h_{1} \geqq \cdots \geqq h_{q} \geqq 0}\left(1-t^{2^{h_{1}}+\cdots+2^{h_{q}}+1}\right)^{-m(q+1)} \\
& \geqq P(X),
\end{aligned}
$$

that is,

$$
\Pi_{n \geqq 1}\left(1-t^{n}\right)^{-b(n)} \geqq P(X)
$$

Let $0 \leqq r<1$. If $\sum_{n \geqq 1} b(n) t^{n}$ converges for $|t| \leqq r$, then $\Pi_{n \geqq 1}\left(1-t^{n}\right)^{b(n)}$ converges and its limit is nonzero. Thus $\Pi_{n \geq 1}\left(1-t^{n}\right)^{-b(n)}$ converges. On the other hand we have that $\log \Pi_{n \geq 1}\left(1-t^{n}\right)^{-b(n)}=\sum_{n \geq 1} b(n)\left\{t^{n}+\right.$ $\cdots\} \geqq \sum_{n \geqq 1} b(n) t^{n} . \quad$ Therefore $\quad \mathrm{r}(P(X)) \geqq \mathrm{r}\left(\Pi_{n \geqq 1}\left(1-t^{n}\right)^{-b(n)}\right)=\min \{1$, $\left.\mathrm{r}\left(\sum b(n) t^{n}\right)\right\}$. So it is sufficient to prove that

$$
\mathrm{r}\left(\sum b(n) t^{n}\right) \geqq \min \left\{1, \mathrm{r} P_{\pi}(X)\right\}
$$

This is proved by means of the following lemmas. Lemma 9 is directly proved by definition. Lemma 10 is proved by induction on $n$ with Lemma 9, (2) and (3).

\section{Lemma 9. (1) $a(n, q)=0$ for $n<q$.}

(2) $a(2 n+1, q)=a(2 n, q-1)$ and $a(2 n+1)=a(2 n)$.

(3) $a(2 n, q)=a(2 n-2, q-2)+a(n, q)$ and $a(2 n)=a(2 n-2)+a(n)$ for $n \geqq 2$.

Lemma 10. If we take a real number $G(C>1)$ and an integer $N$ such that $C^{N} \geqq C^{N-2}+1$, then $a(n) \leqq 2^{N} \cdot C^{n}$ for all $n \geqq 0$.

Now we prove the inequation (8). If we take $C$ and $N$ as in Lemma 10 , then

$$
\begin{aligned}
\mathrm{r}\left(\sum b(n) t^{n}\right) & \geqq \mathrm{r}\left(\sum a(n-1) M(n) t^{n}\right) \\
& \geqq \mathrm{r}\left(\sum 2^{N} \cdot C^{n} M(n) t^{n}\right) \\
& =\mathrm{r}\left(\sum M(n) t^{n}\right) / C \\
& =\min \left\{1, \sum m(n) t^{n}\right\} / C \\
& =\min \left\{1, \mathrm{r} P_{\pi}(X)\right\} / C .
\end{aligned}
$$

Thus

$$
\begin{aligned}
\mathrm{r}\left(\sum b(n) t^{n}\right) & \geqq \sup \left\{\min \left\{1, \mathrm{r} P_{\pi}(X)\right\} / G ; G>1\right\} \\
& =\min \left\{1, \mathrm{r} P_{\pi}(X)\right\}
\end{aligned}
$$


which completes the proof of (8) and of Theorem 3.

Remark. Of course Theorem 3 is valid for odd primes. In this case we can use [4] instead of [3] to prove it.

\section{References}

[1] Bott, R. and Samelson, H., On the Pontrjagin product in spaces of paths, Comm. Math. Helv. 27 (1953), 320-337.

[2] Henn, H. -W., On the growth of homotopy groups, Manuscripta Math. 56 (1986), 235-245.

[3] Serre, J. -P., Cohomologie modulo 2 des complexes d'Eilenberg-MacLane, Comm. Math. Helv. 27 (1953), 198-232.

[4] Umeda, Y., A remark on a theorem of J. -P. Serre, Proc. of Japan Academy, 35 (1959), 563-566. 
